ARTICLE OPEN

\title{
A novel noninvasive method for remote heart failure monitoring: the EuleriAn video Magnification apPLications In heart Failure studY (AMPLIFY)
}

Freddy Abnousi ${ }^{1,2,3}$, Guson Kang ${ }^{1}$, John Giacomini ${ }^{1,4}$, Alan Yeung ${ }^{1}$, Shirin Zarafshar ${ }^{1,4}$, Nicholas Vesom ${ }^{1}$, Euan Ashley (iD), Robert Harrington ${ }^{1}$ and Celina Yong iD $^{1,4}$

Current remote monitoring devices for heart failure have been shown to reduce hospitalizations but are invasive and costly; accurate non-invasive options remain limited. The EuleriAn Video Magnification ApPLications In Heart Failure StudY (AMPLIFY) pilot aimed to evaluate the accuracy of a novel noninvasive method that uses Eulerian video magnification. Video recordings were performed on the neck veins of 50 patients who were scheduled for right heart catheterization at the Palo Alto VA Medical Center. The recorded jugular venous pulsations were then enhanced by applying Eulerian phase-based motion magnification. Assessment of jugular venous pressure was compared across three categories: (1) physicians who performed bedside exams, (2) physicians who reviewed both the amplified and unamplified videos, and (3) direct invasive measurement of right atrial pressure from right heart catheterization. Motion magnification reduced inaccuracy of the clinician assessment of central venous pressure compared to the gold standard of right heart catheterization (mean discrepancy of $-0.80 \mathrm{~cm} \mathrm{H}_{2} \mathrm{O} ; 95 \% \mathrm{Cl}-2.189$ to $0.612, p=0.27$ ) when compared to both unamplified video $\left(-1.84 \mathrm{~cm} \mathrm{H}_{2} \mathrm{O} ; 95 \% \mathrm{Cl}-3.22\right.$ to $\left.-0.46, p=0.0096\right)$ and the bedside exam $\left(-2.90 \mathrm{~cm} \mathrm{H}_{2} \mathrm{O} ; 95 \% \mathrm{Cl}-4.33\right.$ to $1.40, p=0.0002$ ). Major categorical disagreements with right heart catheterization were significantly reduced with motion magnification (12\%) when compared to unamplified video (25\%) or the bedside exam (27\%). This novel method of assessing jugular venous pressure improves the accuracy of the clinical exam and may enable accurate remote monitoring of heart failure patients with minimal patient risk.

npj Digital Medicine (2019)2:80; https://doi.org/10.1038/s41746-019-0159-0

\section{INTRODUCTION}

Heart failure is a tremendously morbid, deadly, and costly disease that affects 6.5 million Americans today. ${ }^{1}$ It is now implicated in one in nine deaths in the United States and at least $20 \%$ of all hospitalizations among persons older than $65 .{ }^{1}$ It costs the United States $\$ 30.7$ billion annually, a figure forecasted to increase to $\$ 70$ billion by $2030 .^{2}$

Much of this economic burden is generated by hospitalizations for heart failure, which represent up to $80 \%$ of direct costs, making heart failure readmissions a key target for cost reduction. ${ }^{2}$ Preventing readmissions requires early provider intervention in the ambulatory setting, but the ideal timing and methods to achieve such interventions remain elusive. A critical step to reducing heart failure hospitalizations is identifying which patients will imminently decompensate by evaluating their volume status. While physicians have traditionally relied on the bedside exam of the jugular venous pressure (JVP) to assess volume status, this can be impractical as a primary method for monitoring outpatients. With the recent explosion of telemedicine, the majority of healthcare institutions in the United States employ some form of virtual interaction, paving the way for monitoring tools that can tap this potential.
Invasive ambulatory hemodynamic monitors have shown promise as early-warning systems in heart failure. A variety of devices have been developed and studied, including right ventricular (COMPASS-HF and REDUCE HF), left atrial (HOMEOSTASIS and LAPTOP-HF), and pulmonary artery (CHAMPION) pressure measurement systems. ${ }^{3-7}$ While devices such as the CardioMEMS monitor have been demonstrated to reduce heart failure readmissions, they may not be scalable: they are invasively implanted, costly upfront (approximately $\$ 17,000$ for a CardioMEMS device), and demand additional personnel and resources to handle the datastream. ${ }^{8}$ Furthermore, their cost-effectiveness remains debatable. ${ }^{8-12}$

In this study, we propose a noninvasive and easily scalable alternative to current invasive remote pressure monitoring systems by combining the bedside examination with modern image processing techniques. Eulerian video magnification is an image processing method by which visually imperceptible periodic motions can be deconstructed and amplified into movements discernible to the naked eye. ${ }^{13}$ We describe the application of Eulerian video magnification to the jugular venous pulse examination and demonstrate its potential as a novel method of noninvasive monitoring of right-sided filling pressures.

\footnotetext{
${ }^{1}$ Division of Cardiovascular Medicine, Department of Medicine, Stanford University Medical Center, Palo Alto, CA, USA; ${ }^{2}$ Yale School of Medicine, Palo Alto, CA, USA; ${ }^{3}$ Yale School of Medicine, New Haven, CT, USA and ${ }^{4}$ Veterans Affairs Palo Alto Medical Center, Palo Alto, CA, USA

Correspondence: Celina Yong (cyong@stanford.edu)

These authors contributed equally: Freddy Abnousi, Guson Kang
}

Received: 20 November 2018 Accepted: 30 July 2019

Published online: 21 August 2019 


\section{RESULTS}

Participant characteristics

Participant characteristics are presented in Table 1. Among the 59 participants enrolled, 48 (81\%) completed the study. The most common reason for study noncompletion was due to cancellation of the right heart catheterization ( $\mathrm{RHC}$ ) procedure (6 patients, $10.2 \%)$. The average age of patients who completed the study was 70 years (SD: 8.04 years); $85 \%$ of participants were over 65 years old. The average BMI was 30 (SD: 4.87) with $83 \%$ of participants qualifying as overweight or obese. Most participants had never smoked $(77 \%)$, while $15 \%$ were former smokers and $8 \%$ were current smokers. Mean chest circumference among this sample was $120 \mathrm{~cm}$ (SD: $12 \mathrm{~cm}$ ).

Invasive vs. non-invasive measurements

A summary of invasive and noninvasive data are reported in Table 2. On average, participant cardiac output and index were normal at $4.94 \mathrm{~L} / \mathrm{min}$ and $2.36 \mathrm{~L} / \mathrm{min} / \mathrm{m}^{2}$, respectively. The average right atrial pressure (RAP) by right heart catheterization was $8.56 \mathrm{~cm} \mathrm{H}_{2} \mathrm{O}$ (IQR 5-10). Similarly, average JVP was $7.11 \mathrm{~cm} \mathrm{H} \mathrm{H}_{2} \mathrm{O}$ (IQR 5-8) when measured at bedside, $7.8 \mathrm{~cm} \mathrm{H}_{2} \mathrm{O}$ (IQR 6.2-8.4) when measured from unamplified video, and $8.8 \mathrm{~cm} \mathrm{H}_{2} \mathrm{O}$ (IQR 7.2-10) when measured from motion-amplified video.

All noninvasive modalities yielded JVPs lower than the corresponding invasive right atrial pressures, though the magnitude varied depending on the modality (Table 3 ). When measured at the bedside, JVP was on average $2.90 \mathrm{~cm} \mathrm{H}_{2} \mathrm{O}$ lower than corresponding right heart catheterization measurements $(95 \% \mathrm{Cl}$ -4.33 to $1.40, p=0.0002$ ). This discrepancy narrowed when measured via unamplified video $\left(-1.84 \mathrm{~cm} \mathrm{H}_{2} \mathrm{O} ; 95 \% \mathrm{Cl}-3.22\right.$ to $-0.46, p=0.0096)$. After motion magnification, this discrepancy was no longer significant $\left(-0.80 \mathrm{~cm} \mathrm{H}_{2} \mathrm{O} ; 95 \% \mathrm{Cl}-2.189\right.$ to 0.612 , $p=0.27$ ).

To better understand the practical clinical utility of the video magnification, we compared the noninvasive to the invasive measurements by characterizing them qualitatively. Each invasive and noninvasive measurement was designated "normal" if it was less than or equal to $7 \mathrm{~cm} \mathrm{H}_{2} \mathrm{O}$, "borderline" if greater than 7 but less than or equal to $10 \mathrm{~cm} \mathrm{H} \mathrm{H}_{2} \mathrm{O}$, or "elevated" if greater than $10 \mathrm{~cm} \mathrm{H}_{2} \mathrm{O}$. "Agreement" with right heart catheterization was

\begin{tabular}{|ll|}
\hline Table 1. Patient demographics & \\
\hline & Study completed $(N=48)$ \\
\hline Age, Mean \pm SD (IQR) & $69.9 \pm 8.04(67-73)$ \\
Age brackets, $n(\%)$ & $7(14.6)$ \\
$40-64$ years & $31(64.6)$ \\
$65-74$ years & $10(20.8)$ \\
$75+$ years & $29.6 \pm 4.87(26.3-32.3)$ \\
BMI, Mean \pm SD (IQR) & \\
BMI brackets, $n(\%)$ & $1(2.1)$ \\
Underweight & $7(14.6)$ \\
Normal weight & $17(35.4)$ \\
Overweight & $23(47.9)$ \\
Obese & \\
Smoker, $n$ (\%) & $37(77.1)$ \\
No & $7(14.6)$ \\
Former & $4(8.3)$ \\
Yes & $119.6 \pm 12.01(112.5-129)$ \\
Chest circumference (cm), Mean \pm SD (IQR) & \\
\hline$B M I$ body mass index & \\
\hline
\end{tabular}

achieved if both categories were the same (e.g., both were "elevated"); partial disagreement was noted if there was a onecategory discrepancy (e.g., one was "normal" and the other was "borderline"); and complete disagreement was noted if there was a two-category discrepancy (e.g., one was "normal" and the other was "elevated"). These pooled data are presented in Fig. 1. Complete disagreement was found in $27 \%$ of bedside assessments, $25 \%$ of unamplified video assessments, and only $12 \%$ of amplified video assessments. Neither bedside exam nor unamplified video assessments skewed in a statistically significant fashion towards "agreement" or "disagreement" with right heart catheterization ( $p=0.44$ and $p=0.10$, respectively; Table 4$)$. In contrast, assessments made with amplified video more often agreed or partially disagreed $(p=0.0034)$ than the other noninvasive modalities.

Table 2. JVP and RHC measurements. Cardiologist mean pressure measurements are reported in $\mathrm{cm} \mathrm{H}_{2} \mathrm{O}$ except as otherwise noted

\begin{tabular}{|c|c|c|}
\hline & \multicolumn{2}{|c|}{ Study completed $(N=48)$} \\
\hline & $N$ & Mean \pm SD (IQR) \\
\hline \multicolumn{3}{|l|}{$\mathrm{RHC}$} \\
\hline Cardiac output & 48 & $4.94 \pm 0.92 \mathrm{~L} / \mathrm{min}(4.25-5.50)$ \\
\hline Cardiac index & 48 & $2.36 \pm 0.34 \mathrm{~L} / \mathrm{min} / \mathrm{m}^{2}(2.18-2.56)$ \\
\hline Heart rate & 48 & $65.73 \pm 12.53$ beats $/ \min (59-73.25)$ \\
\hline PAP (mean) & 48 & $21.02 \pm 7.47 \mathrm{mmHg}(16-24)$ \\
\hline PCWP & 48 & $15.17 \pm 7.97 \mathrm{mmHg}(9.75-19.5)$ \\
\hline RAP & 48 & $8.56 \pm 5.09 \mathrm{~cm} \mathrm{H}_{2} \mathrm{O}(5-10)$ \\
\hline \multicolumn{3}{|l|}{ Bedside physician exams } \\
\hline JVP: 1st physician exam & 48 & $7.06 \pm 2.85(5-8)$ \\
\hline JVP: 2nd physician exam & 48 & $6.52 \pm 3.14(5-7)$ \\
\hline JVP (mean) & 48 & $7.11 \pm 3.21(5-8)$ \\
\hline \multicolumn{3}{|l|}{ Videos: Unamplified } \\
\hline JVP: Cardiologist 1 & 48 & $7 \pm 2(5-10)$ \\
\hline JVP: Cardiologist 2 & 48 & $9 \pm 2(7-11)$ \\
\hline JVP: Cardiologist 3 & 48 & $9 \pm 3(7-11)$ \\
\hline JVP: Cardiologist 4 & 48 & $8.3 \pm 3.4(6-10)$ \\
\hline JVP: Cardiologist 5 & 48 & $7 \pm 1(6-8)$ \\
\hline JVP: Cardiologist 6 & 48 & $9 \pm 3(6-10)$ \\
\hline JVP: Cardiologist 7 & 48 & $5 \pm 4(2-5)$ \\
\hline JVP: Cardiologist 8 & 48 & $6 \pm 3(5-7)$ \\
\hline JVP: Cardiologist 9 & 48 & $10 \pm 2(8-10)$ \\
\hline JVP (mean) & 48 & $7.8 \pm 2(6.2-8.4)$ \\
\hline \multicolumn{3}{|l|}{ Videos: Amplified } \\
\hline JVP: Cardiologist 1 & 48 & $7 \pm 3(5-10)$ \\
\hline JVP: Cardiologist 2 & 48 & $9 \pm 2(7-11)$ \\
\hline JVP: Cardiologist 3 & 48 & $11 \pm 3(8-13)$ \\
\hline JVP: Cardiologist 4 & 48 & $9.4 \pm 3.8(7-11)$ \\
\hline JVP: Cardiologist 5 & 48 & $8 \pm 2(7-9)$ \\
\hline JVP: Cardiologist 6 & 48 & $10 \pm 3(8-12)$ \\
\hline JVP: Cardiologist 7 & 48 & $8 \pm 4(5-10)$ \\
\hline JVP: Cardiologist 8 & 48 & $7 \pm 3(5-9)$ \\
\hline JVP: Cardiologist 9 & 48 & $11 \pm 3(9-12)$ \\
\hline JVP (mean) & 48 & $8.8 \pm 2.2(7.2-10)$ \\
\hline
\end{tabular}

$J V P$ jugular venous pressure, $R H C$ right heart catheterization, $R A P$ right atrial pressure, $P A P$ pulmonary artery pressure, $P C W P$ pulmonary capillary wedge pressure, IQR interquartile range 
Table 3. Comparison of means between clinically and invasively measured jugular venous pressure

\begin{tabular}{llll}
\hline Measurement differences & Mean difference $\left(\mathrm{cm} \mathrm{H}_{2} \mathrm{O}\right)$ & $95 \% \mathrm{Cl}$ for mean difference & Wilcoxon signed rank $p$ value \\
\hline Bedside exam vs. $\mathrm{RHC}$ & -2.90 & -4.33 to -1.40 & 0.0002 \\
Unamplified video vs. RHC & -1.84 & -3.22 to -0.46 & 0.0096 \\
Amplified video vs. $\mathrm{RHC}$ & -0.80 & -2.18 to 0.61 & 0.27 \\
\hline Cl confidence interval, $\mathrm{RHC}$ right heart catheterization & & \\
\hline
\end{tabular}

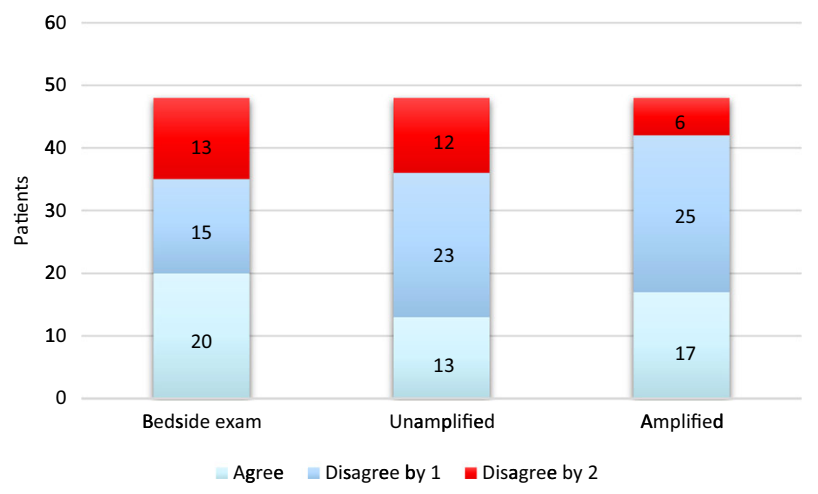

Fig. 1 Agreement with right heart catheterization by assessment type. "Agreement" with right heart catheterization was achieved if clinicians characterized the JVP similarly (e.g., both were "elevated"); "disagree by 1" was noted where a one-category discrepancy existed (e.g., one was "normal" and the other was "borderline"); and "disagree by 2" was noted if there was a two-category discrepancy (e.g., one was "normal" and the other was "elevated")

Table 4. Accuracy of JVP characterization compared to right heart catheterization

\begin{tabular}{|c|c|c|c|c|}
\hline Category $^{a}$ & Observed & Expected & Pearson's $x^{2}$ & $p$ value \\
\hline \multicolumn{5}{|l|}{ Bedside exam } \\
\hline Agree & 20 & 16 & \multirow[t]{3}{*}{1.625} & \multirow[t]{3}{*}{0.44} \\
\hline Disagree by 1 & 15 & 16 & & \\
\hline Disagree by 2 & 13 & 16 & & \\
\hline \multicolumn{5}{|l|}{ Unamplified } \\
\hline Agree & 13 & 16 & \multirow[t]{3}{*}{4.625} & \multirow[t]{3}{*}{0.10} \\
\hline Disagree by 1 & 23 & 16 & & \\
\hline Disagree by 2 & 12 & 16 & & \\
\hline \multicolumn{5}{|l|}{ Amplified } \\
\hline Agree & 17 & 16 & \multirow[t]{3}{*}{11.375} & \multirow[t]{3}{*}{0.0034} \\
\hline Disagree by 1 & 25 & 16 & & \\
\hline Disagree by 2 & 6 & 16 & & \\
\hline \multicolumn{5}{|c|}{$\begin{array}{l}\text { JVP jugular venous pressure } \\
\text { a"Agreement" achieved if the assessment categorization was the same as } \\
\text { right heart catheterization (e.g., both "borderline"); "disagree by 1" noted if } \\
\text { there was a one-category discrepancy (e.g., "normal" and "borderline"); } \\
\text { "disagree by 2" noted if there was a two-category discrepancy (e.g., } \\
\text { "normal" and "elevated"). Expected values assume a random frequency } \\
\text { distribution across categories } \\
\text { Bold value indicate statistical significance }\end{array}$} \\
\hline
\end{tabular}

\section{DISCUSSION}

The AMPLIFY pilot study suggests that computerized motion amplification can improve the accuracy of clinical bedside JVP measurement. Historically, studies of the bedside exam have reported variable accuracy, and some suggest that it tends to underestimate the central venous or right atrial pressure. ${ }^{14,15}$ Our data confirm this tendency. Furthermore, our data suggest that the bedside exam is inaccurate at characterizing right-sided filling pressures: $27 \%$ of characterizations made at the bedside by two independent cardiologists were completely erroneous, which may reflect the high proportion of obesity in this study. Despite the challenging population, motion magnification resulted in less central venous pressure discrepancy with right heart catheterization as compared to bedside or unamplified video assessments. Perhaps more relevantly, it reduced significant categorical disagreements with right heart catheterization compared to the other noninvasive modalities.

Since its first description in 2012, Eulerian phase-based motion amplification has been re-tooled for a variety of purposes, such as measuring heart rate by amplifying the imperceptible color shifts of the skin with each pulse ${ }^{13}$ or localizing and labeling major blood vessels during surgeries in real time. ${ }^{16}$ This method is the first published application of Eulerian phase-based motion amplification to heart failure diagnostics.

Perhaps most importantly, the AMPLIFY ambulatory heart failure monitoring strategy has the potential to expand modern telehealth capabilities. Telehealth adoption has been estimated to exceed $60 \%$ across all U.S. healthcare institutions, and virtual interactions (e.g. video, telephone, e-mail) already outnumber inperson visits in some healthcare systems. ${ }^{17}$ With that in mind, we designed AMPLIFY to emulate the telehealth setting: all video assessments were completed by cardiologists who never made physical contact with study participants. Unlike current invasive monitoring tools, which generate overwhelming amounts of data that do not integrate into existing electronic health record systems, the on-demand nature of motion-amplified monitoring should yield a more manageable datastream, generated at clinically important time points. Finally, as a software-based technique, motion amplification has great potential for scalability as it requires no specialized equipment beyond what is already available in many telehealth ecosystems. Given our use of commercially available video equipment, we speculate that this technology could also be eventually adapted for use with video equipment available on most modern mobile phones.

AMPLIFY has several limitations. As a pilot study, the cohort is small and derived from a predominantly male Veterans Affairs population, and the results should not be generalized beyond the scope of the study. AMPLIFY was intentionally conducted on an outpatient population to emulate the telehealth setting and therefore does not apply to inpatient monitoring. The study was not designed to examine clinical outcomes such as heart failure decompensations or cost-effectiveness. From a technical standpoint, the method itself is reliant on detecting changes in local contrast and may have theoretical limitations when applied to patients with darker skin tones, though our study did capture a variety of skin tones. Finally, the clinical assessment of JVP in general can be inaccurate when it is very high (e.g., above the earlobe); as such, we do not consider it a quantitative technique in its current form.

The next steps will be to refine, automate, and implement this novel technique in the telemedicine setting to compare it with modern invasive monitoring systems on a larger scale. Visual 


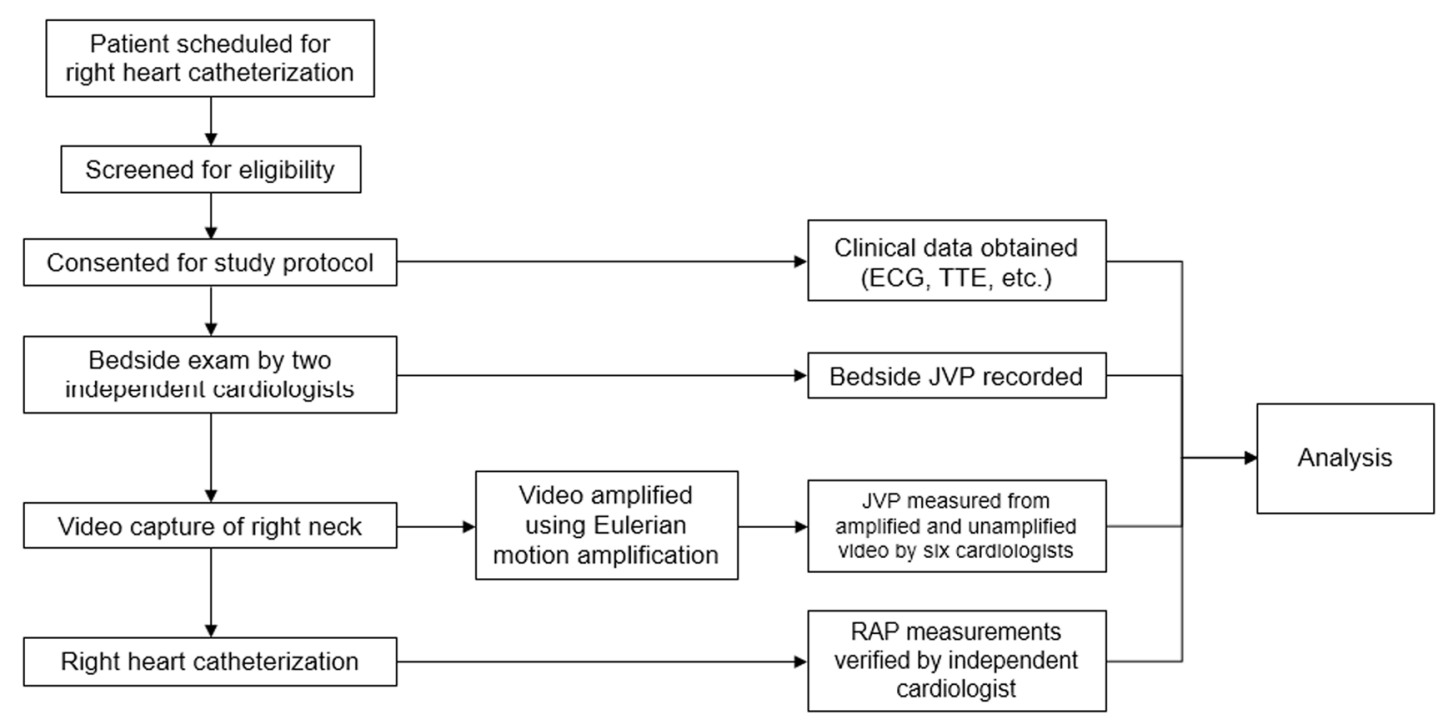

Fig. 2 AMPLIFY protocol. ECG electrocardiogram, TTE transthoracic echocardiogram, JVP jugular venous pressure, RAP right atrial pressure

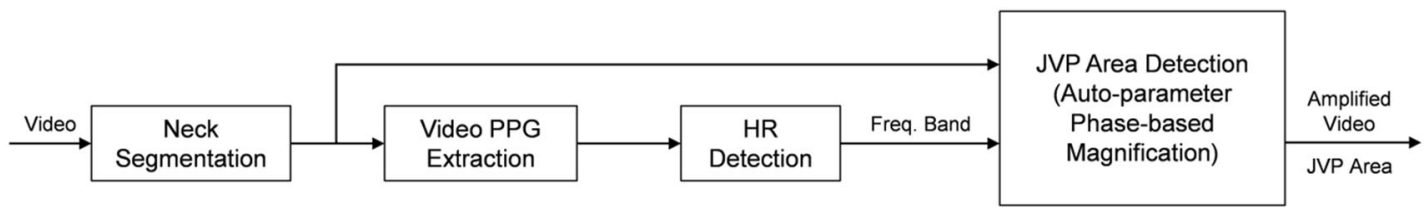

Fig. 3 Video stream magnification procedure. PPG photoplethysmogram

amplification techniques are heavily reliant on image contrast, and improvements to acquisition technique, such as using off-camera point light sources to improve local contrast and signal-to-noise yield, may improve accuracy. This may be especially relevant to patients with darker skin tones and naturally lower tonal contrast. Furthermore, standardizing acquisition position, scale, and distance from the patient, as well as identification of landmarks from which JVP is clinically measured (e.g., clavicle or the sternal angle) are potential targets for optimization. In the future, advances in mobile hardware capabilities may improve clinical usability by allowing for real-time visualization of the jugular venous pulse (socalled "augmented reality"). Finally, automation of the measurement process using computer vision techniques could further decrease the need for human supervision and improve costefficiency.

In conclusion, AMPLIFY serves as a pilot to demonstrate the potential utility and scalability of motion magnification for providing clinically relevant remote monitoring of patients with heart failure.

\section{METHODS}

The AMPLIFY study was a pilot clinical trial evaluating the efficacy of using video image processing as a novel method for measuring JVP. This study was approved by both the Stanford Institutional Review Board and the Veterans Affairs Research and Development Board. Written informed consent was obtained from each participant.

\section{Participants}

Eligible participants were screened through the VA electronic medical record system. Patients were eligible for inclusion if they were over 18 years of age and were scheduled to undergo right heart catheterization for any indication at the Palo Alto Veterans Affairs Hospital. Exclusion criteria included existing right internal jugular vascular access, ventilatory support or airway/cervical deformity interfering with neck visualization, inability to tolerate lying at a 30 or 45 -degree angle, or inability to lie still for the duration of the video recording.

\section{Procedure}

The AMPLIFY protocol is outlined in Fig. 2. After potential participants were screened for eligibility, informed consent was obtained and participants were assigned an anonymous study identification number.

Using commercially available video and lighting equipment, two 60-s video clips of the right side of the participant's neck were acquired: one with the participant lying on a standard wedge pillow at a $45^{\circ}$ angle, and the second while sitting at a $90^{\circ}$ angle. During all video acquisition, the patient was monitored with a continuous three-lead electrocardiogram (right upper chest, left upper chest, one leg) with a commercially available portable ECG machine (Heal Force PC-80B). The acquisition system consisted of a commercial digital camera connected to a laptop PC. The camera system consisted of a Basler aCA1920-155uc (Sony IMX174 global shutter CMOS sensor) with a Kowa LM25HC 1" 25 mm F1.4 lens. A batterypowered LED light source was mounted above the camera facing the same direction to provide illumination. A laptop computer running custom software streamed uncompressed, $1920 \times 1280$, 8-bit RGB at 155 frames per second to a solid-state drive. The acquisition system was stabilized on a tripod cart to reduce artifact from translational and rotational vibrations.

Two cardiologists independently performed bedside examinations of each participant's JVP and recorded their measurements prior to right heart catheterization. Clinicians qualitatively assessed venous pressure by estimating the orthogonal height of the JVP column, which appears as a subtle, transient movement of the skin. Copies of the pre-catheterization ECG and the participant's most recent echocardiogram report within the last year were collected. Upon completion of right heart catheterization, copies of all pressure tracings were obtained, from which pressure measurements were extracted by an independent cardiologist.

The de-identified video recordings were then sent to Google LLC for image processing. The video streams were processed as shown in Fig. 3. First, the neck region was segmented using a weighted Gaussian mixture model. A video photoplethysmogram (VPPG) was then extracted to determine the heart rate, which was used in the later motion magnification block to set the frequency bands of interest.

Eulerian phase-based video magnification was then used to amplify the pulsations of the right internal jugular vein as described in Wu et al..$^{13}$ and Wadhwa et al. ${ }^{18}$ The basic principle of the approach is to decompose a standard video sequence into a time series of color values at a given pixel. This time series is then filtered for a prespecified band of temporal 


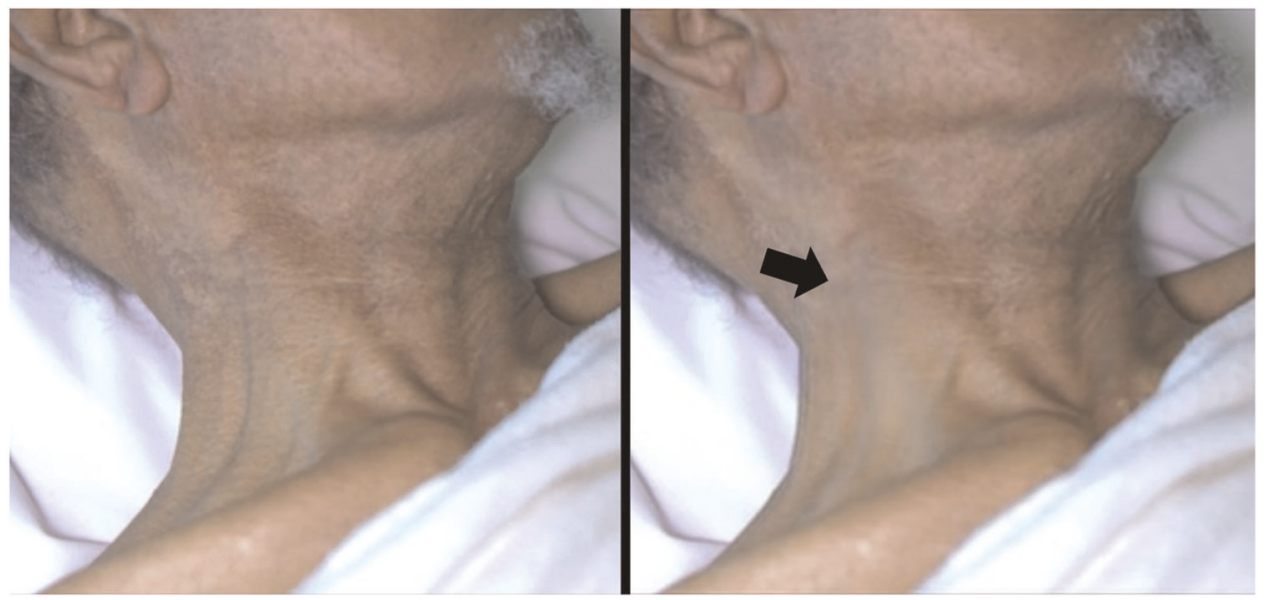

Fig. 4 Video magnification screen capture. Still screen capture from unamplified video (left) and amplified video (right). Though easier to appreciate while in motion (Supplemental Videos 1 and 2), exaggerated pulsatile distension of the internal jugular vein is visible as a motion blur-like artifact up to the black arrow. Written informed consent allowing publication of the participant's images was obtained

frequencies (in this application, a range including normal resting human heart rates); the resultant signal is then amplified by a specified magnification factor and reconstituted back into video format. Our code was built off the publicly available MATLAB code; signal processing was performed by commercially available personal computers. A sample video is included in the supplementary materials (Supplementary Video 1 demonstrates unamplified and amplified video); a representative screen capture from the video is shown in Fig. 4.

After image processing, both the unamplified and amplified videos were assessed by nine cardiologists. The cardiologists were blinded with respect to all patient data, including the right heart catheterization results, and were not involved with any other part of the study. The cardiologists were instructed to approximate the JVP based on both the unamplified and amplified video streams without the use of measuring aids to reflect realworld practice; scale was inferred visually using anatomic landmarks.

Measures. Demographic (age, smoking history) and anthropometric (height, weight, chest circumference) data were collected from each participant during the study visit. Participants' body mass indices were calculated from the height and weight and categorized using the CDC definitions for underweight $(<18.5)$, normal weight (18.6-24.9), overweight (25-29.9), and obese ( $\geq 30)$.

Each bedside cardiologist's assessment included an estimation of the JVP at bedside (reported in centimeters of water) and the angle at which it was assessed (Supplementary Table 1). Data obtained from the right heart catheterization tracings included pressure values for the right atrium, right ventricle, pulmonary capillary wedge pressure, pulmonary artery, as well as cardiac output, cardiac index, intraprocedural heart rate, and a record of the sedative medications given. Cardiologist assessment of the unamplified and amplified jugular vein videos reported estimated JVP in centimeters of water (Supplementary Table 2).

To be included in the final analysis, participants were required to have completed (1) right heart catheterization, (2) bedside assessment, and (3) video assessment.

\section{Statistical analysis}

JVP assessments from the noninvasive techniques and RHC measurements were descriptively summarized for all participants who completed the study. The average of the bedside JVP assessments was calculated for each participant to obtain an averaged JVP to compare against the RHC RAP. The average JVP assessed by video (unamplified and amplified) was also compared against the RHC RAP. To translate the measurements to clinically actionable categories, we converted them to "normal," "borderline," or "elevated" if they were less than or equal to $7 \mathrm{~cm} \mathrm{H}_{2} \mathrm{O}$, greater than 7 but less than or equal to $10 \mathrm{~cm} \mathrm{H}_{2} \mathrm{O}$, or greater than $10 \mathrm{~cm} \mathrm{H}_{2} \mathrm{O}$, respectively. ${ }^{19-22}$ Mean differences and $95 \% \mathrm{Cl}$ of the mean difference was calculated between each of the averaged JVPs and RHC right atrial pressure. All analyses were conducted using SAS 9.3 (Cary, NC).

\section{Reporting summary}

Further information on research design is available in the Nature Research Reporting Summary linked to this article.

\section{DATA AVAILABILITY}

The hemodynamic data that support the findings of this study are anonymized and presented in the Supplementary Information. Additional video data that support the findings of this study are available on request from the corresponding author (C.Y.). The video data are not publicly available to minimize any exposure of potentially sensitive material.

\section{CODE AVAILABILITY}

The code employed to process the video streams is publicly available at http:// people.csail.mit.edu/mrub/vidmag/\#code.

\section{ACKNOWLEDGEMENTS}

The study was funded by Google Inc., Palo Alto, CA.

\section{AUTHOR CONTRIBUTIONS}

Co-first authorship is shared between Drs. Kang and Abnousi. All authors listed have been directly involved in study design, data interpretation, critical revision, and final approval of this manuscript. Furthermore, Drs. Kang, Abnousi, and Yong were involved in manuscript preparation. All authors accept accountability for the accuracy and integrity of the work.

\section{ADDITIONAL INFORMATION}

Supplementary information accompanies the paper on the npj Digital Medicine website (https://doi.org/10.1038/s41746-019-0159-0).

Competing interests: F.A. previously received consulting fees from Google Inc. The remaining authors declare no competing interests.

Publisher's note: Springer Nature remains neutral with regard to jurisdictional claims in published maps and institutional affiliations.

\section{REFERENCES}

1. Benjamin, E. J. et al. Heart disease and stroke statistics - 2017 update: a report from the American Heart Association. Circulation 135, e146-e603 (2017).

2. Heidenreich, P. A. et al. Forecasting the impact of heart failure in the United States: a policy statement from the American Heart Association. Circ. Heart Fail. 6, 606-619 (2013). 
3. Bourge, R. C. et al. Randomized controlled trial of an implantable continuous hemodynamic monitor in patients with advanced heart failure: the COMPASS-HF study. J. Am. Coll. Cardiol. 51, 1073-1079 (2008).

4. Adamson, P. B. et al. Continuous hemodynamic monitoring in patients with mild to moderate heart failure: results of The Reducing Decompensation Events Utilizing Intracardiac Pressures in Patients With Chronic Heart Failure (REDUCEhf) trial. Congest. Heart Fail. 17, 248-254 (2011).

5. Ritzema, J. et al. Physician-directed patient self-management of left atrial pressure in advanced chronic heart failure. Circulation 121, 1086-1095 (2010).

6. Maurer, M. S. et al. Rationale and design of the left atrial pressure monitoring to optimize heart failure therapy study (LAPTOP-HF). J. Card. Fail. 21, 479-488 (2015).

7. Givertz, M. M. et al. Pulmonary artery pressure-guided management of patients with heart failure and reduced ejection fraction. J. Am. Coll. Cardiol. 70, 1875-1886 (2017).

8. Sandhu, A. T. \& Heidenreich, P. A. Heart failure management with ambulatory pulmonary artery pressure monitoring. Trends Cardiovasc. Med. 28, 212-219 (2018).

9. Sandhu, A. T. et al. Cost-effectiveness of implantable pulmonary artery pressure monitoring in chronic heart failure. JACC Heart Fail. 4, 368-375 (2016).

10. Schmier, J. K., Ong, K. L. \& Fonarow, G. C. Cost-effectiveness of remote cardiac monitoring with the CardioMEMS heart failure system. Clin. Cardiol. 40, 430-436 (2017).

11. Cowie, M. R., Simon, M., Klein, L. \& Thokala, P. The cost-effectiveness of real-time pulmonary artery pressure monitoring in heart failure patients: a European perspective. Eur. J. Heart Fail. 19, 661-669 (2017).

12. Martinson, M., Bharmi, R., Dalal, N., Abraham, W. T. \& Adamson, P. B. Pulmonary artery pressure-guided heart failure management: US cost-effectiveness analyses using the results of the CHAMPION clinical trial. Eur. J. Heart Fail. 19, 652-660 (2017).

13. $\mathrm{Wu}, \mathrm{H}$.-Y. et al. Eulerian video magnification for revealing subtle changes in the world. ACM Trans. Graph. 31, 1-8 (2012).

14. Elder, A., Japp, A. \& Verghese, A. How valuable is physical examination of the cardiovascular system? BMJ 354, i3309 (2016).

15. Stein, J. H., Neumann, A. \& Marcus, R. H. Comparison of estimates of right atrial pressure by physical examination and echocardiography in patients with congestive heart failure and reasons for discrepancies. Am. J. Cardiol. 80, 1615-1618 (1997).

16. Amir-Khalili, A. et al. Auto localization and segmentation of occluded vessels in robot-assisted partial nephrectomy. Med. Image Comput. Comput. Assist. Inter. 17, 407-414 (2014).

17. Tuckson, R. V., Edmunds, M. \& Hodgkins, M. L. Telehealth. N. Engl. J. Med. 377, 1585-1592 (2017)

18. Wadhwa, N., Rubinstein, M., Durand, F. \& Freeman, W. T. Phase-based video motion processing. ACM Trans. Graph. 32, 1 (2013).

19. Butman, S. M., Ewy, G. A., Standen, J. R., Kern, K. B. \& Hahn, E. Bedside cardiovascular examination in patients with severe chronic heart failure: importance of rest or inducible jugular venous distension. J. Am. Coll. Cardiol. 22, 968-974 (1993).

20. Ewy, G. A. \& Marcus, F. L. Bedside estimation of venous pressure. Heart Bull. 17, 41-44 (1965).

21. Ewy, G. A. in Signs and Symptoms in Cardiology (eds L. D. Horowitz \& B. M. Groves) Ch. 132, 55 (JB Lippincott, Philadelphia, 1985).

22. Kern, M. J., Lim, M. J. \& Goldstein, J. A. Hemodynamic Rounds: Interpretation of Cardiac Pathophysiology from Pressure Waveform Analysis (Wiley-Blackwell, Hoboken, NJ, 2009).

(C) Open Access This article is licensed under a Creative Commons Attribution 4.0 International License, which permits use, sharing, adaptation, distribution and reproduction in any medium or format, as long as you give appropriate credit to the original author(s) and the source, provide a link to the Creative Commons license, and indicate if changes were made. The images or other third party material in this article are included in the article's Creative Commons license, unless indicated otherwise in a credit line to the material. If material is not included in the article's Creative Commons license and your intended use is not permitted by statutory regulation or exceeds the permitted use, you will need to obtain permission directly from the copyright holder. To view a copy of this license, visit http://creativecommons. org/licenses/by/4.0/.

(c) This is a U.S. government work and not under copyright protection in the U.S.; foreign copyright protection may apply 2019 